\title{
Preoperative peripheral blood neutrophil-to-lymphocyte ratios (NLR) and platelet-to-lymphocyte ratio (PLR) related nomograms predict the survival of patients with limited-stage small-cell lung cancer
}

\author{
Chunji Chen ${ }^{1 \#}$, Haitang Yang ${ }^{1 \#}$, Deng Cai ${ }^{1 \#}$, Lujie Xiang ${ }^{2 \#}$, Wentao Fang ${ }^{1}$, Rui Wang ${ }^{1}$ \\ ${ }^{1}$ Department of Thoracic Surgery, Shanghai Chest Hospital, Shanghai Jiao Tong University, Shanghai, China; ${ }^{2}$ Nursing Department, Shanghai \\ Chest Hospital, Shanghai Jiao Tong University, Shanghai, China \\ Contributions: (I) Conception and design: R Wang, W Fang; (II) Administrative support: R Wang; (III) Provision of study materials or patients: C \\ Chen, R Wang, W Fang; (IV) Collection and assembly of data: C Chen, H Yang, D Cai; (V) Data analysis and interpretation: C Chen, H Yang, D \\ Cai; (VI) Manuscript writing: All authors; (VII) Final approval of manuscript: All authors. \\ \#These authors contributed equally to this work. \\ Correspondence to: Rui Wang; Wentao Fang. Department of Thoracic Surgery, Shanghai Chest Hospital, Shanghai Jiao Tong University, 241 West \\ Huaihai Road, Shanghai 200030, China. Email: vwtfang@hotmail.com; rui_wang788@163.com.
}

Background: We aim to establish neutrophil-to-lymphocyte ratio (NLR) and platelet-to-lymphocyte ratio (PLR) related nomograms based on the clinical data and peripheral blood markers to predict the survivals of patients with limited-stage small-cell lung cancer (LS-SCLC).

Methods: A total of 299 LS-SCLC patients after surgery were enrolled in this study. Univariate and multivariate analyses were conducted to select independent prognostic factors to develop the nomograms and then subjected to bootstrap internal validation. The optimal cutoff value of NLR and PLR before surgery was calculated by X-tile (version 3.6.1) and the overall survival (OS) was analyzed by Kaplan-Meier method and compared by log-rank test.

Results: According to the X-tile calculation, the NLR value and PLR cutoff values are 2.6 and 156.7, respectively. The prognosis of patients with elevated NLR or PLR value was significantly worse than patients with lower NLR (HR =1.798, 95\% CI: 1.284-2.518, P=0.001) or PLR (HR =1.781, 95\% CI: 1.318-2.407, $\mathrm{P}<0.001)$ value. Two Nomograms were developed according to the two multivariate cox regression models based on NLR and PLR. Concordance index (C-index) curves and calibration curves show that the two models have a better effect in predicting prognosis. At the same time, compared with the tumor node metastasis (TNM) staging system, our models also show better accuracy and stability.

Conclusions: Elevated NLR and PLR predict poor prognosis in their respective nomograms in patients with LS-SCLC.

Keywords: Limited-stage small-cell lung cancer (LS-SCLC); neutrophil-to-lymphocyte ratio (NLR); platelet-tolymphocyte ratio (PLR); prognosis

Submitted Aug 31, 2020. Accepted for publication Dec 29, 2020.

doi: $10.21037 /$ tlcr-20-997

View this article at: http://dx.doi.org/10.21037/tlcr-20-997 


\section{Introduction}

Small cell lung cancer (SCLC) represents approximately $15 \%$ of all lung cancers (1). It is characterized by rapid progress and prone to distant metastasis. Although SCLC is highly sensitive to chemotherapy and radiotherapy, the prognosis of most patients with this disease is still poor. A systematic analysis shows that the 5-year OS rate of small cell lung cancer is only between $10 \%$ and $15 \%$ (2).

Inflammation plays a key role in the occurrence and development of tumors, and the development of cancer is easily regulated by immune cells (3). Neutrophil-tolymphocyte ratio (NLR) and platelet-to-lymphocyte ratio (PLR) are considered to be potential systemic inflammatory markers and is of great significance to predict the survival and prognosis of various malignant solid tumors (4-12). However, the role of inflammatory markers in SCLC is rarely studied $(13,14)$. The immunotherapy of programmed death receptor-1 (PD-1) antibody changed the mode of SCLC treatment, and these checkpoint inhibitors showed better results than standard therapy, the results from two large clinical studies (IMpower133 and CASPIAN) show that immunotherapy can significantly improve the prognosis of patients with SCLC $(15,16)$. Intriguingly, recent studies have shown that NLR and PLR have predictive value for checkpoint inhibitors in the treatment of non-small cell lung cancer (NSCLC), elevated NLR and PLR indicate poor prognosis of immunotherapy (17-20). Exploring the effect of NLR and PLR on the prognosis of patients with SCLC may provide reference significance for the selection of patient subgroups in immunotherapy.

In this study, we explored the relationship between inflammatory markers and survival of limited-stage smallcell lung cancer (LS-SCLC) patients, and attempted to evaluate the prognostic value of NLR and PLR in SCLC, hoping to provide clues for immunotherapy in patients with SCLC.

We present the following article in accordance with the TRIPOD reporting checklist (available at http://dx.doi. org/10.21037/tlcr-20-997).

\section{Methods}

\section{Patients}

All procedures performed in this study involving human participants were in accordance with the Declaration of Helsinki (as revised in 2013). The study was approved by the institutional review board of Shanghai Chest Hospital
(No. KS(Y)1755). Because of the study's retrospective nature, the need for written informed consent was waived. From January 2006 and January 2014, a total of 438 patients with pathologically-confirmed SCLC who underwent complete surgical resection as initial treatment for primary tumor were collected. The study flow chart is shown in Figure 1. Finally, A total of 299 patients met the inclusion criteria.

Hematological indexes were routinely examined within one week before operation. SCLC staging was performed according to the 8 th tumor node metastasis (TNM) classification, and overall survival (OS) was defined as the date of diagnosis until the date of death or last followup visit. The records of neutrophil, lymphocyte and platelet count from peripheral blood tests prior to surgery (within 1 week) were retrieved. We also collected data on preoperative serum tumor markers including CA125, CA19-9, carcinoembryonic antigen (CEA), neuron-specific enolase (NSE) and squamous cell carcinoma antigen (SCCA) levels. The expression of common neuroendocrine markers in the resected tumor samples [NSE; SYN (synaptophysin); CGA (chromogranin A) and CD56] was confirmed by immunohistochemistry.

\section{Statistical analysis}

Chi-square test and the Student's $t$-test were used to compare the categorical and continuous variables, respectively. Univariable and multivariable Cox regression analyses were performed to predict the independent risk factors of OS. The curves of OS, as well as the comparisons, were calculated by Kaplan-Meier survival curves and the log-rank test. The optimal truncation value of NLR and PLR affecting the prognosis was determined by using $\mathrm{X}$-tile software (version 3.6.1) and the minimum $P$ value method (21).

Based on the results of multivariate analysis, the nomograms are established and subjected to 1,000 bootstrap resamples for internal validation of the primary training cohort. Concordance index (C-index) was used to evaluate the model performance of the prediction results. By comparing the predicted survival rate and the observed survival rate after bias correction, the calibration plot curves of the nomograms for 1-, 3- and 5 -years OS were performed.

All the data were analyzed by SPSS 23.0 software package (SPSS Inc., Chicago, IL, USA), unless otherwise indicated. And the $\mathrm{C}$-index curves and calibration curves were drawn by $\mathrm{R}$ software (version 3.6.1; R Foundation for 


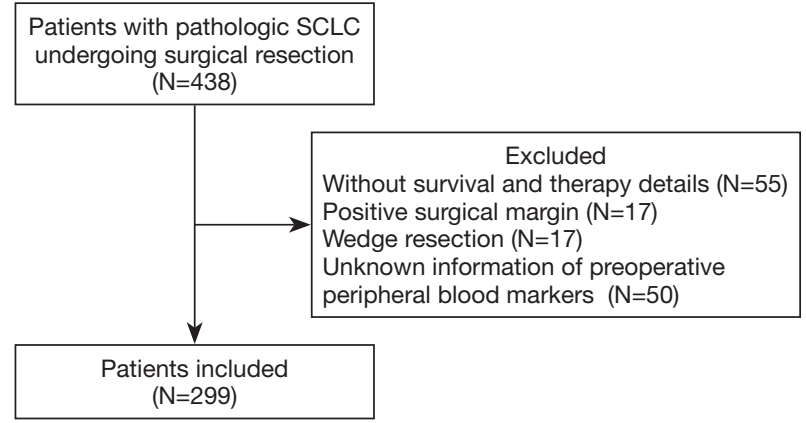

Figure 1 Flow diagram of patient selection process.

Table 1 Demographic and tumor data of all patients

\begin{tabular}{|c|c|}
\hline Characteristic & No. (\%) \\
\hline Total & 299 \\
\hline \multicolumn{2}{|l|}{ Age, years } \\
\hline Mean \pm SD & $59.4 \pm 8.6$ \\
\hline \multicolumn{2}{|l|}{ Gender } \\
\hline Male & $255(85.3)$ \\
\hline Female & $44(14.7)$ \\
\hline \multicolumn{2}{|l|}{ Smoking status } \\
\hline No & $60(20.1)$ \\
\hline Yes & $239(79.9)$ \\
\hline \multicolumn{2}{|c|}{ Preoperative serum NSE abnormality } \\
\hline No & $239(79.9)$ \\
\hline Yes & $60(20.1)$ \\
\hline \multicolumn{2}{|l|}{ Other serum TM positive } \\
\hline No & $212(70.9)$ \\
\hline Yes & $87(29.1)$ \\
\hline \multicolumn{2}{|c|}{ Neuroendocrine markers by IHC } \\
\hline None positive NE marker & $11(3.7)$ \\
\hline 1 positive NE marker & $69(23.1)$ \\
\hline$\geq 2$ positive NE markers & $141(47.1)$ \\
\hline Unknown & $78(26.1)$ \\
\hline \multicolumn{2}{|l|}{ Surgery type } \\
\hline Lobectomy & $237(79.3)$ \\
\hline Pneumonectomy & $34(11.4)$ \\
\hline Segmentectomy & $28(9.4)$ \\
\hline \multicolumn{2}{|l|}{ Histology } \\
\hline Pure SCLC & $196(65.6)$ \\
\hline Combined SCLC & $103(34.4)$ \\
\hline
\end{tabular}

Table 1 (continued)
Table 1 (continued)

\begin{tabular}{|c|c|}
\hline Characteristic & No. (\%) \\
\hline \multicolumn{2}{|l|}{ NLR } \\
\hline Median (range) & $2.13(0.57-8.38)$ \\
\hline \multicolumn{2}{|l|}{ PLR } \\
\hline Median (range) & $115.5(40-420)$ \\
\hline \multicolumn{2}{|l|}{ Tumor size } \\
\hline Mean \pm SD & $3.8 \pm 1.6$ \\
\hline \multicolumn{2}{|l|}{ Tumor Stage } \\
\hline I & $67(22.4)$ \\
\hline II & $94(31.4)$ \\
\hline III & $138(46.2)$ \\
\hline \multicolumn{2}{|l|}{ Adjuvant } \\
\hline No & $24(8.0)$ \\
\hline Yes & $275(92.0)$ \\
\hline \multicolumn{2}{|c|}{ Cycles of chemotherapy } \\
\hline$\geq 4$ & $251(83.8)$ \\
\hline $0-4$ & $48(16.1)$ \\
\hline \multicolumn{2}{|c|}{ Postoperative RT to chest } \\
\hline No & $104(34.8)$ \\
\hline Yes & $195(65.2)$ \\
\hline \multicolumn{2}{|l|}{$\mathrm{PCl}$} \\
\hline No & $200(66.9)$ \\
\hline Yes & $99(33.1)$ \\
\hline \multicolumn{2}{|l|}{ PET scan } \\
\hline No & $78(26.1)$ \\
\hline Yes & $221(73.9)$ \\
\hline
\end{tabular}

$\mathrm{PCl}$, prophylactic cranial irradiation; SCLC, small cell lung cancer; RT, radiation therapy; SD, standard deviation; IHC, immunohistochemistry; NSE, neuron specific enolase; NLR, neutrophil-to-lymphocyte ratio; PLR, platelet-to-lymphocyte ratio; $\mathrm{PET}$, positron emission tomography.

Statistical Computing). All tests were 2-tailed; $\mathrm{P}$ value $<0.05$ was considered statistically significant.

\section{Results}

The characteristic information of patients included in the study were shown in Table 1; Of the 299 patients included in the study, there were 255 males $(85.3 \%)$ and 44 females $(14.7 \%)$, with an average age of 59.4 years 
Table 2 Univariable and multivariate cox regression analysis of factors associated with overall survival

\begin{tabular}{|c|c|c|c|c|c|c|c|c|c|}
\hline \multirow{2}{*}{ Variable } & \multicolumn{3}{|c|}{ Univariable } & \multicolumn{6}{|c|}{ Multivariable } \\
\hline & $P$ & $\mathrm{HR}$ & $95 \% \mathrm{Cl}$ & $\mathrm{P}$ & $\mathrm{HR}$ & $95 \% \mathrm{Cl}$ & $P$ & $\mathrm{HR}$ & $95 \% \mathrm{Cl}$ \\
\hline Age, years & 0.339 & 1.007 & $0.991-1.023$ & & & & & & \\
\hline Gender & 0.237 & 1.254 & $0.862-1.823$ & & & & & & \\
\hline NLR & $<0.001$ & 1.215 & $1.095-1.350$ & 0.001 & 1.798 & $1.284-2.518$ & - & - & - \\
\hline PLR & 0.004 & 1.004 & $1.001-1.006$ & - & - & - & $<0.001$ & 1.781 & $1.318-2.407$ \\
\hline NSE positive & 0.067 & 1.352 & $0.979-1.866$ & 0.382 & 1.172 & $0.821-1.674$ & 0.395 & 1.164 & $0.820-1.653$ \\
\hline Other TM positive & 0.615 & 1.080 & $0.801-1.456$ & & & & & & \\
\hline Surgery type & 0.224 & 1.139 & $0.923-1.406$ & & & & & & \\
\hline Histology & 0.179 & 0.817 & $0.608-1.097$ & & & & & & \\
\hline Pathologic tumor size & 0.014 & 1.105 & $1.021-1.197$ & 0.225 & 1.055 & $0.967-1.151$ & 0.147 & 1.066 & $0.978-1.163$ \\
\hline p-stage & $<0.001$ & 1.399 & $1.169-1.675$ & $<0.001$ & 1.817 & $1.459-2.262$ & $<0.001$ & 1.559 & $1.273-1.909$ \\
\hline Adjuvant & $<0.001$ & 0.485 & $0.340-0.692$ & 0.400 & 1.345 & $0.675-2.679$ & 0.271 & 1.471 & $0.739-2.929$ \\
\hline Cycles of chemotherapy & 0.003 & 0.911 & $0.858-0.968$ & $<0.001$ & 0.374 & $0.225-0.621$ & $<0.001$ & 0.284 & $0.172-0.468$ \\
\hline Postoperative RT to chest & 0.038 & 0.736 & $0.551-0.983$ & 0.016 & 0.680 & $0.497-0.931$ & 0.006 & 0.642 & $0.466-0.883$ \\
\hline $\mathrm{PCl}$ & 0.010 & 0.765 & $0.501-0.911$ & 0.006 & 0.651 & $0.481-0.882$ & 0.002 & 0.625 & $0.461-0.847$ \\
\hline
\end{tabular}

NLR, neutrophil-to-lymphocyte ratio; PLR, platelet-to-lymphocyte ratio; NSE, neuron specific enolase; PET, positron emission tomography; $\mathrm{PCl}$, prophylactic cranial irradiation.

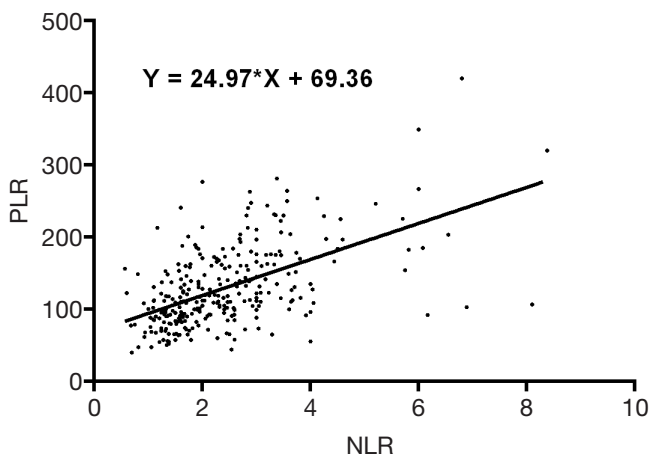

Figure 2 Scatter plots of NLR and PLR. NLR, neutrophil-tolymphocyte ratio; PLR, platelet-to-lymphocyte ratio.

(33-79 years). Adjuvant chemotherapy regimens are shown in Table S1, all of which are platinum-containing. Moreover, postoperative radiation therapy to chest was performed in 195 (65.2) patients, and prophylactic cranial irradiation (PCI) was performed in $99(33.1 \%)$ patients.

\section{Univariate and multivariate analysis}

In univariate analysis, NLR $(\mathrm{P}<0.001)$, PLR $(\mathrm{P}=0.004)$, pathologic tumor size $(\mathrm{P}=0.014), \mathrm{p}$-Stage $(\mathrm{P}<0.001)$, adjuvant therapy $(\mathrm{P}<0.001)$, cycles of chemotherapy $(\mathrm{P}=0.003)$, Postoperative RT to $\operatorname{chest}(\mathrm{P}=0.003)$ and $\mathrm{PCI}$ $(\mathrm{P}=0.010)$ were associated with OS of patients (Table 2). The factors included in the final multivariate Cox regression analysis were unadjusted factors closely related to survival and progress in univariate analysis $(\mathrm{P}<0.10)$. Considering that there is a strong collinearity between NLR and PLR (Figure 2), we established two cox regression models based on NLR and PLR, respectively. Finally, in the two multivariate analysis models, NLR and PLR were both significant predictors for OS.

\section{NLR and PLR analyses}



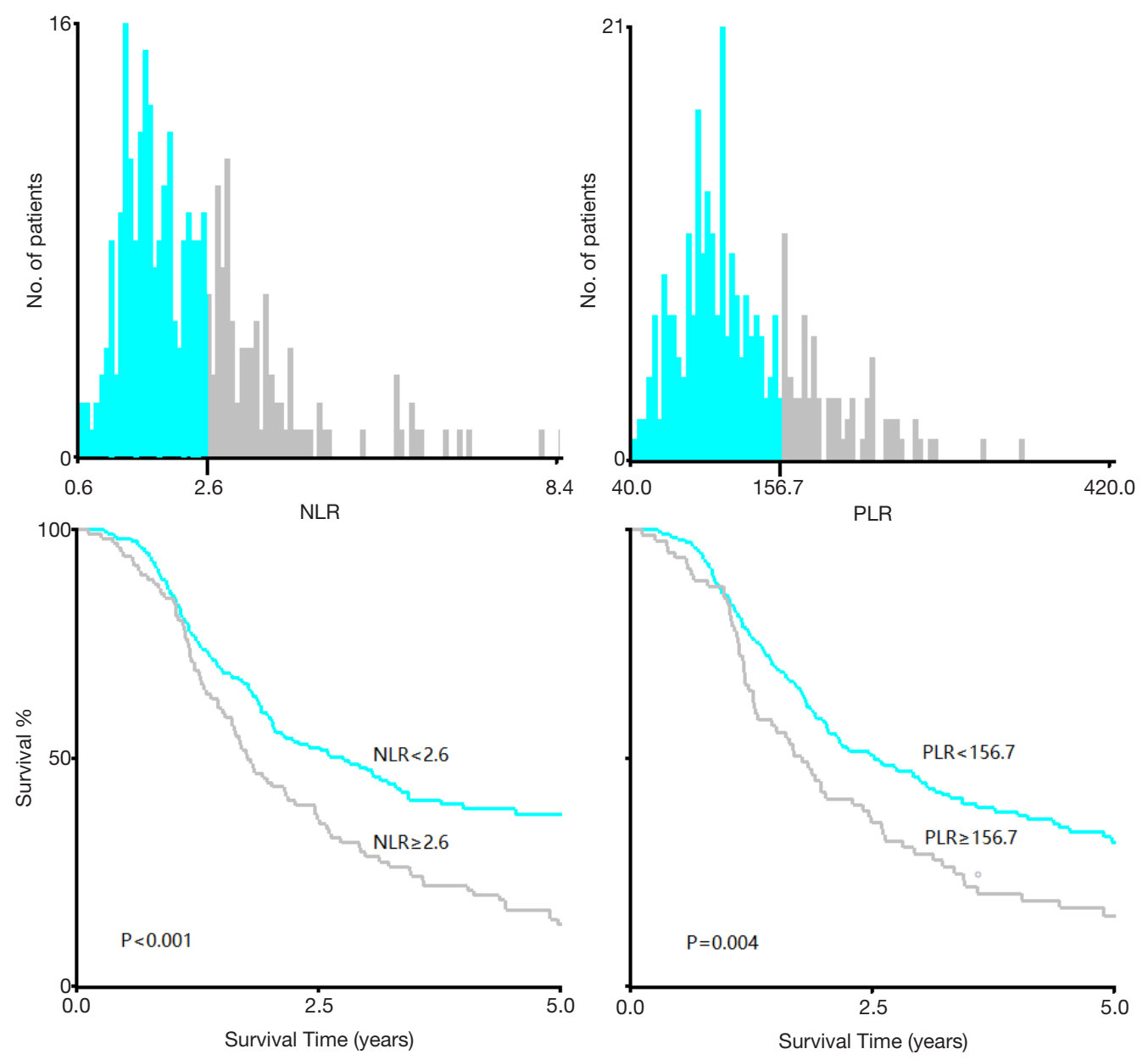

Figure 3 X-Tile analysis of survival based on NLR and PLR. The cutoff value of NLR and PLR are 2.6 and 156.7, respectively. NLR, neutrophil-to-lymphocyte ratio; PLR, platelet-to-lymphocyte ratio.

The mean NLR and PLR level were $2.13(0.57-8.38)$ and 115.5 (40.0-420), respectively. According to the results of multivariate analysis of model 1 and model 2, NLR and PLR were independent risk factors for OS in their respective models, so we tried to find their optical cutoff value to predict the prognosis. The optimal truncation value of NLR and PLR affecting the prognosis was determined by using $\mathrm{X}$-tile software (version 3.6.1) and the minimum $\mathrm{P}$ value method. The best cutoffs of NLR (2.6) and PLR (156.7) were defined as those with the minimum $\mathrm{P}$ value of log-rank test in the X-tile. (Figure 3). Patients with higher pretreatment NLR $(\geq 2.6)$ had worse OS time than patients with lower pretreatment total lymphocyte count $(<2.6$; $\mathrm{P}<0.001)$. Similarly, patients with higher pretreatment PLR $(\geq 156.7)$ had worse OS performance than patients with lower pretreatment PLR $(<156.7 ; \mathrm{P}=0.004)$.
Table 3 summarizes the clinical baseline information of patients between NLR groups and PLR groups. There was no difference in NLR and PLR levels among groups with different variables, except for the stage. Patients with a higher tumor stage tend to have higher NLR values. Subgroup analysis according to the tumor stage was performed and the results are shown in Figure 4. The results showed that the elevated NLR and PLR had poor prognosis in each stage.

\section{Nomograms development and validation}

We established 2 nomograms based on Model 1 and Model 2 , respectively, including significant prognostic factors in multivariate analysis, and were able to reflect 1-, 3- and 5 -year OS (Figure 5). The nomograms included important 
Table 3 Comparison of baseline data of patients grouped by NLR and PLR cutoff values (total: $\mathrm{n}=299$ )

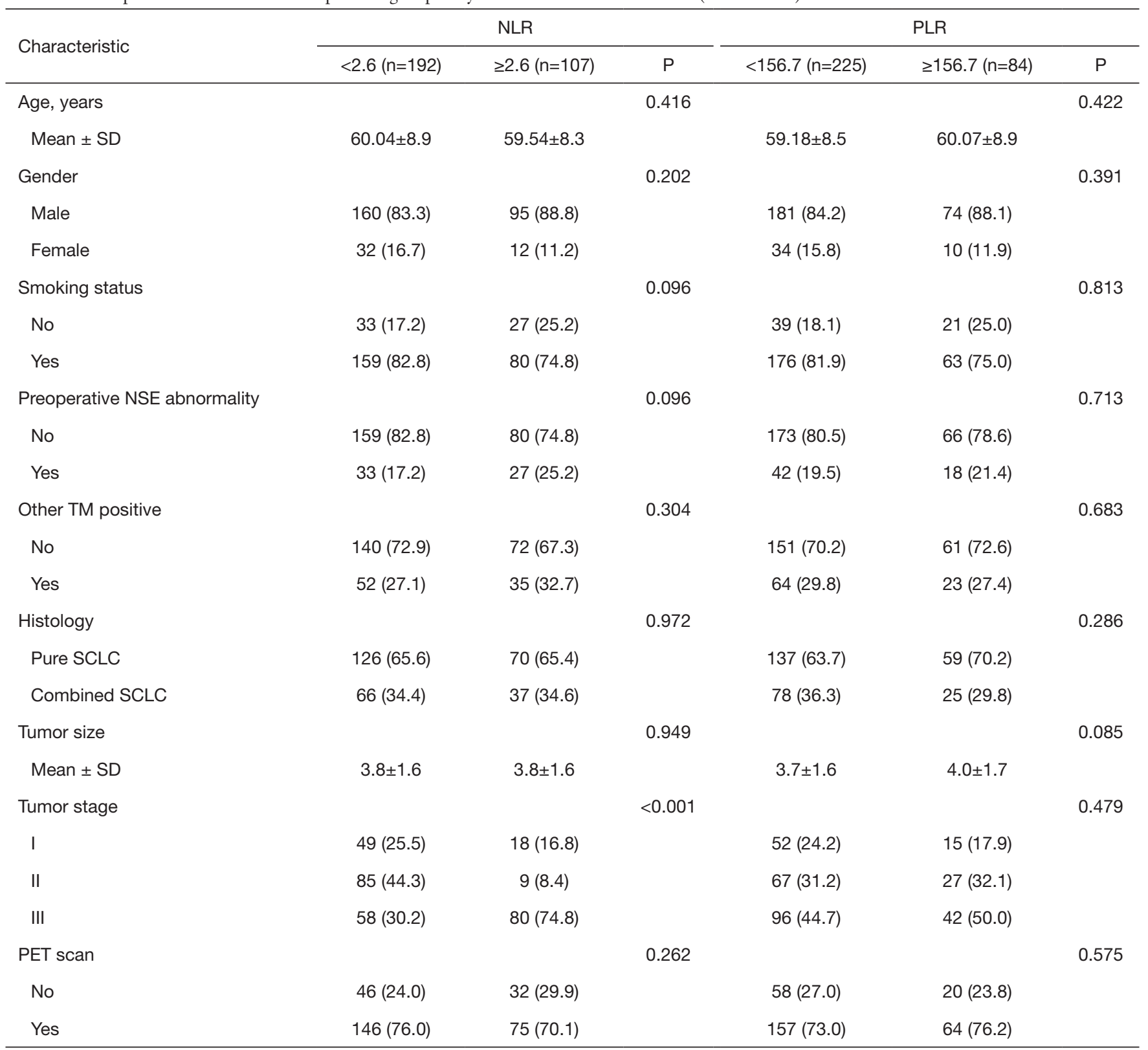

NLR, neutrophil-to-lymphocyte ratio; PLR, platelet-to-lymphocyte ratio; NSE, neuron specific enolase; SCLC, small-cell lung cancer; PET, positron emission tomography.

prognostic factors, such as NLR, PLR, pathological stages, cycles of chemotherapy, postoperative RT to chest and PCI. Nomograms showed that NLR and PLR contribute significantly to survival.

The comparison of the C-index of Model 1 and Model 2 after 1,000 bootstrap internal validation was shown in Figure 6. We found that there is little difference in the degree of differentiation between the two models, and both of them show good prediction capacity. The calibration curves of these two Models show acceptable consistency between the prediction of 1-year, 3-year and 5-year OS and the actual observations (Figure 7).

The C-index of the two models was compared with that of the TNM staging system. After bias correction, the results showed that both model 1 and model 2 were significantly better than the TNM staging system in 

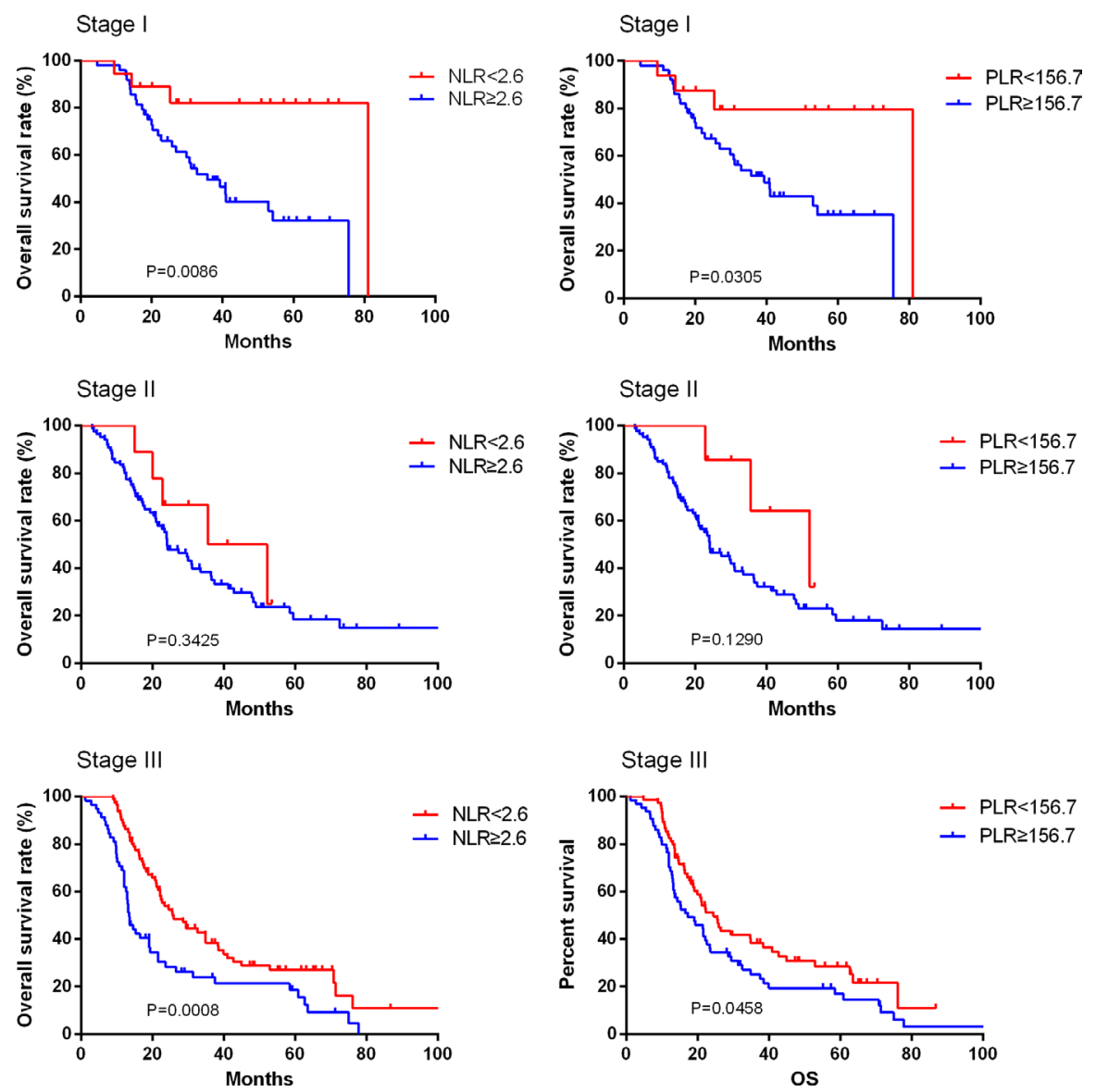

Figure 4 Survival curve of patients with stage I, II and III according to NLR and PLR dichotomy. NLR, neutrophil-to-lymphocyte ratio; PLR, platelet-to-lymphocyte ratio.

predicting the prognosis of patients (Figure 8). The calibration curves of 1-, 3- and 5-year OS show that the stability of the two models is better than that of the TNM staging system (Figure 9).

\section{Discussion}

SCLC is a tumor with a high malignant degree and poor prognosis, representing approximately $15 \%$ of all lung cancers worldwide. Despite the high response to chemotherapy, most patients still died of postoperative recurrence. Immunotherapy is a rising means of tumor therapy in recent years, and clinical trials on immunotherapy for SCLC are being carried out. Results from two large clinical studies (IMpower133 and CASPIAN) show that immunotherapy can significantly improve the prognosis for patients with SCLC $(15,16)$. Recently, studies have shown that NLR and PLR are closely related to the efficacy of immune checkpoint inhibitors in patients with NSCLC (17-20). Amaral et al. (17) revealed that elevated pretreatment NLR and PLR were independent predictors of decreased PFS and OS for patients with locally advanced and metastatic non-small cell lung cancer treated with Nivolumab and Pembrolizumab. Diem et al. (22) also revealed that the elevated value of NLR and PLR before treatment was associated with shorter OS and PFS and lower response rate in patients with metastatic NSCLC treated with nivolumab. Although PD-L1 antibodies or tumor mutation burden (TMB) are the most common biomarkers in immunotherapy, their stability and reliability are still controversial. Compared with expensive PD-L1 antibody tests or TMB tests, it is meaningful to evaluate additional, cheap and easily available prognostic markers to initially identify patients with SCLC who can benefit from immunotherapy.

The data in this study were based on our previous 

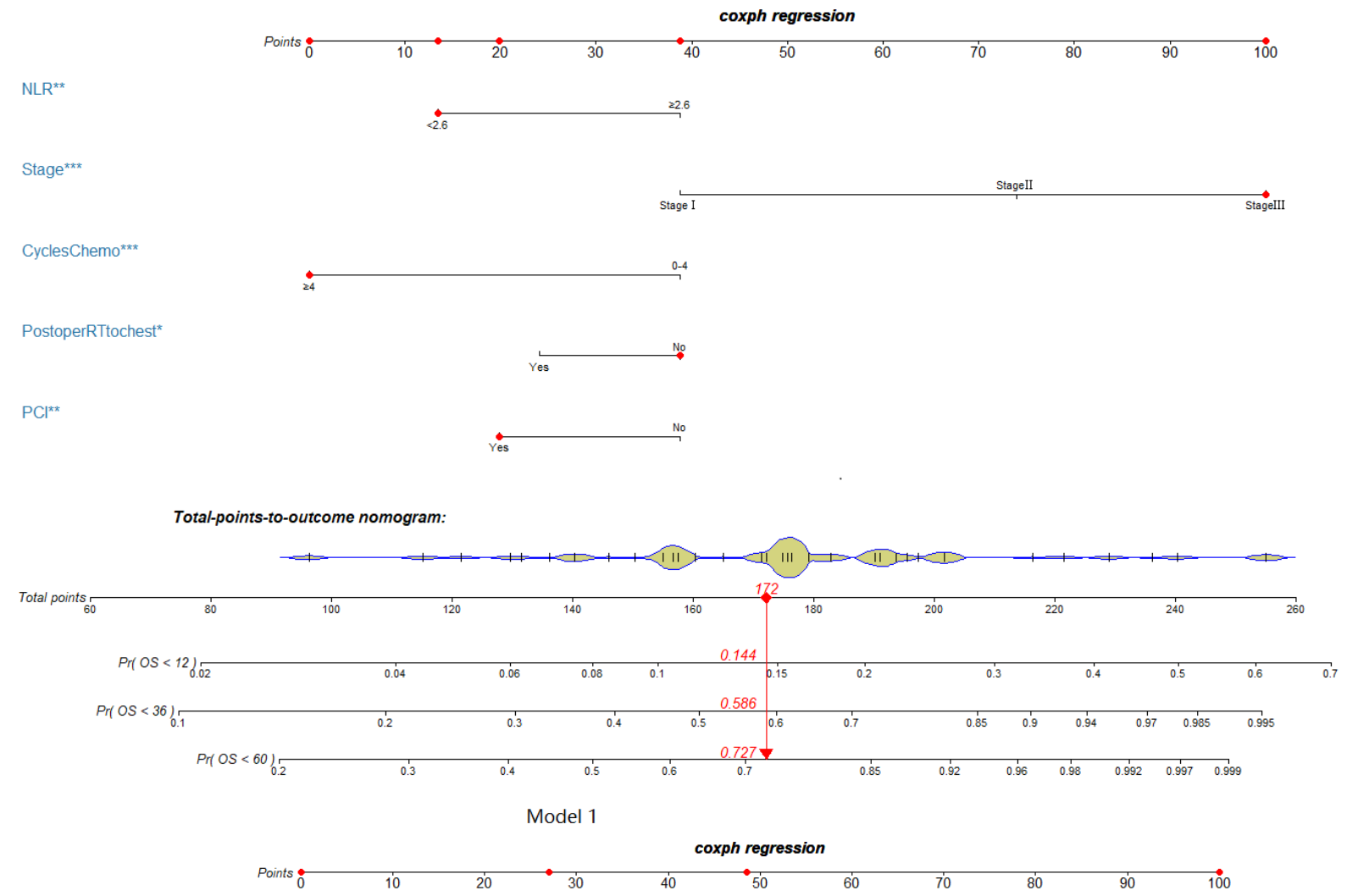

$\operatorname{PLR}{ }^{* \star *}$

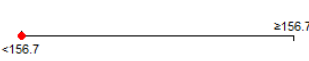

Stage $e^{* * *}$

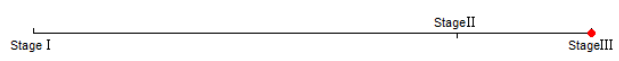

CyclesChemo $0^{\star \star *}$

PostoperRTtochest*

(1)

$\left.\mathrm{PCl}\right|^{* *}$
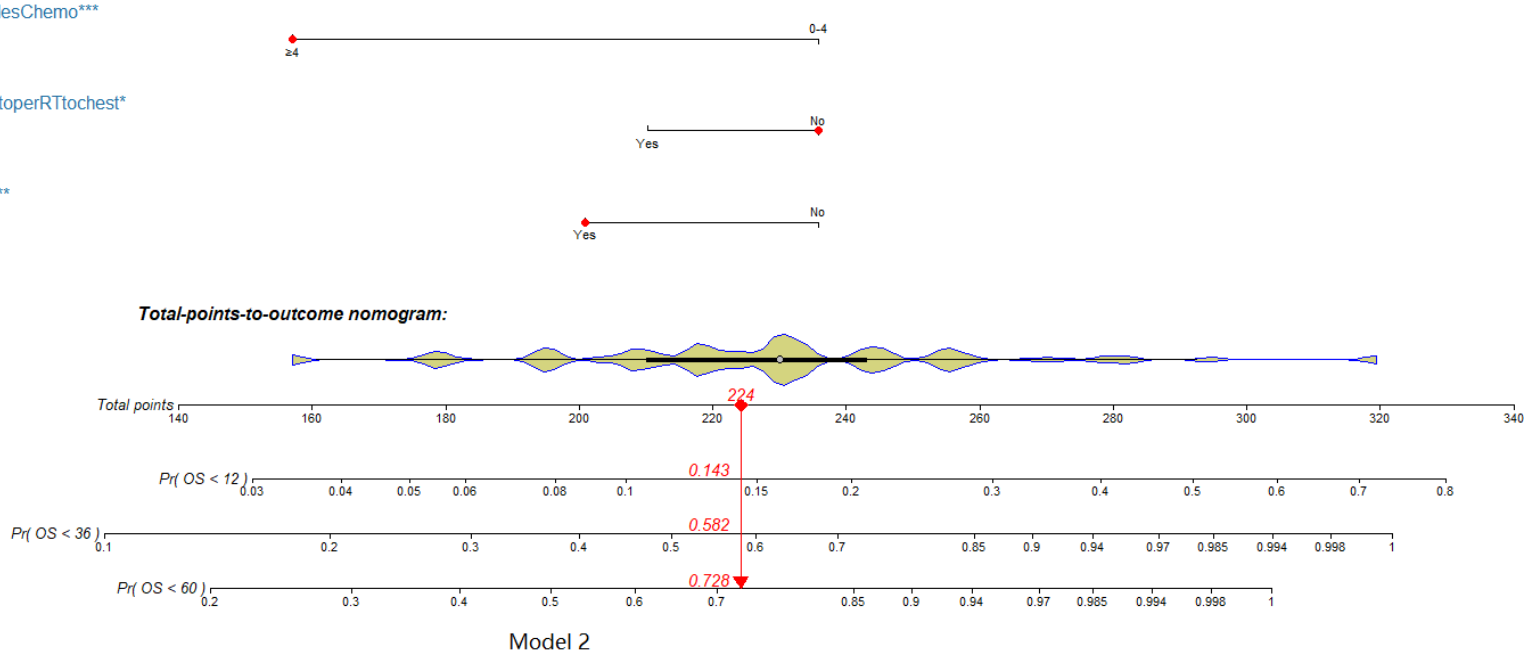

Figure 5 Postoperative prognostic nomograms based on Model 1 and Model 2 of multivariate analysis for patients with resected small-cell lung cancer. *, $\mathrm{P}<0.05 ;{ }^{* *}, \mathrm{P}<0.01 ;{ }^{* * *}, \mathrm{P}<0.001$. 


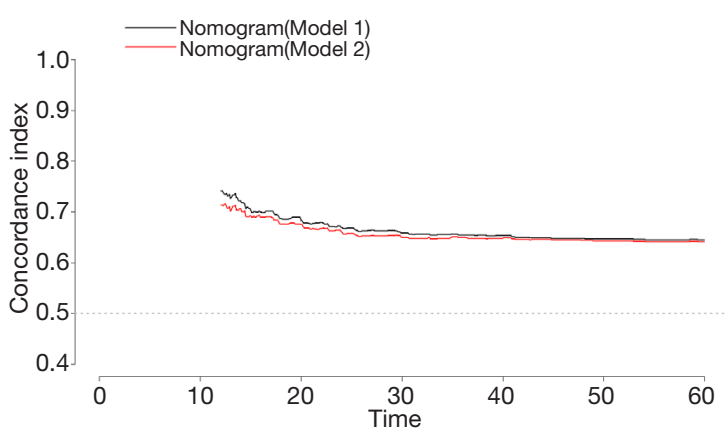

Figure 6 C-index curves of Model 1 and Model 2 in the primary cohort after 1,000 bootstrap internal validation.
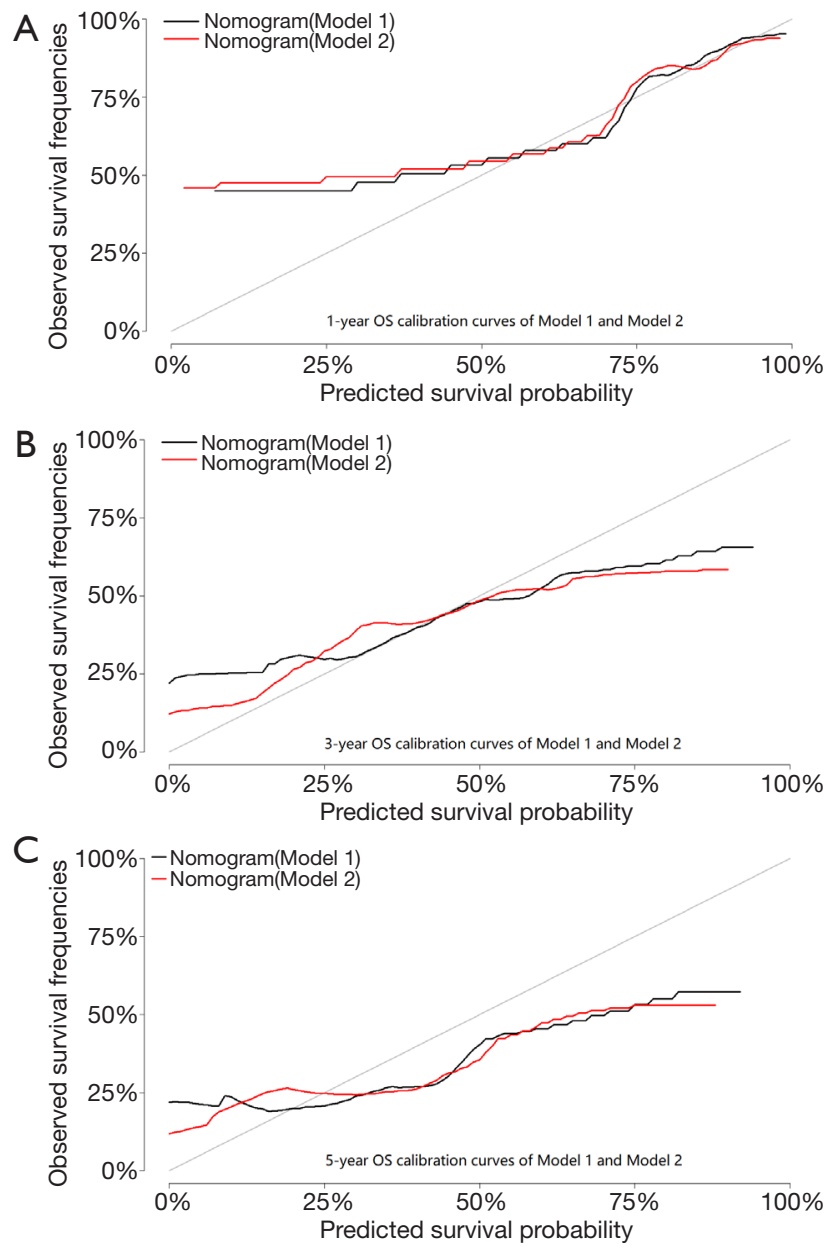

Figure 7 The calibration curve for predicting patient survival of (A) 1-, (B) 3- and (C) 5-year OS in the primary cohort after 1,000 bootstrap internal validation. OS, overall survival.

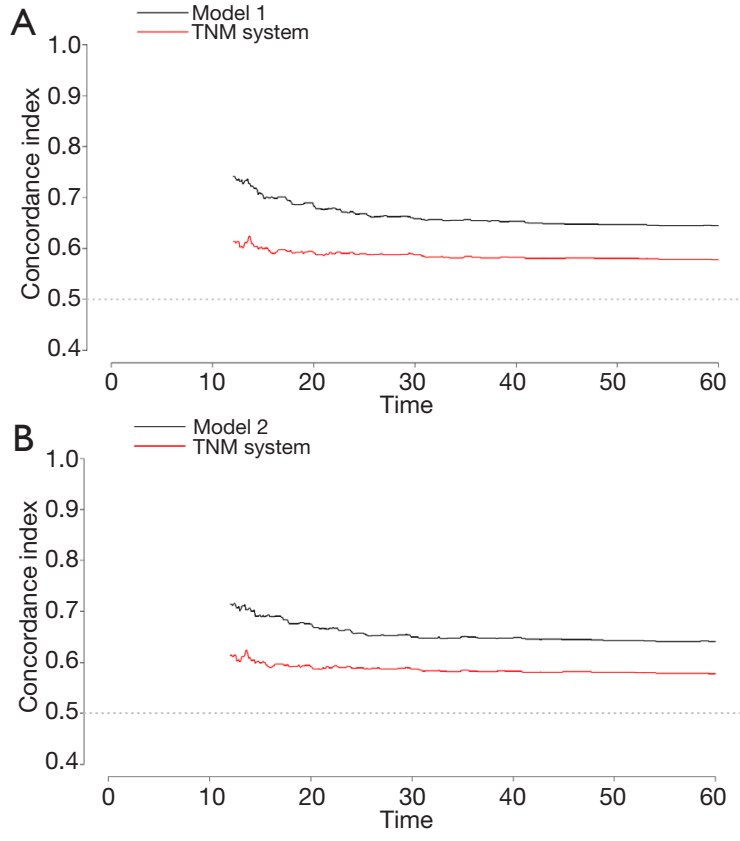

Figure 8 The C-index curves of the TNM staging system compared with model 1 (A) and model 2 (B) after 1,000 bootstraps. TNM, tumor node metastasis.

studies of patients with surgically resected SCLC (23-25). In this study, we established two nomograms for predicting prognosis based on NLR and PLR, respectively, and validated the model internally. At the same time, we also compared it with the TNM staging system. The results show that our model has good stability and accuracy.

To our knowledge, this is the first report on the relationship between NLR or PLR and the prognosis of patients with SCLC based on Chinese data. Xie et al. (13) reported the effects of NLR and PLR on the prognosis of patients with advanced and limited small cell lung cancer based on Mayo Clinic data and the results shown that elevated PLR $(\mathrm{P}<0.001)$ and NLR $(\mathrm{P}<0.001)$ were significantly associated with a worse prognosis in patients with SCLC, which was consistent with our research results. But their data lack some common clinical indicators of SCLC, such as levels of neuroendocrine markers such as NSE, CGA, and preoperative tumor markers. And our research contains this part of the data, although the abnormal tumor markers was not associated with prognosis. 

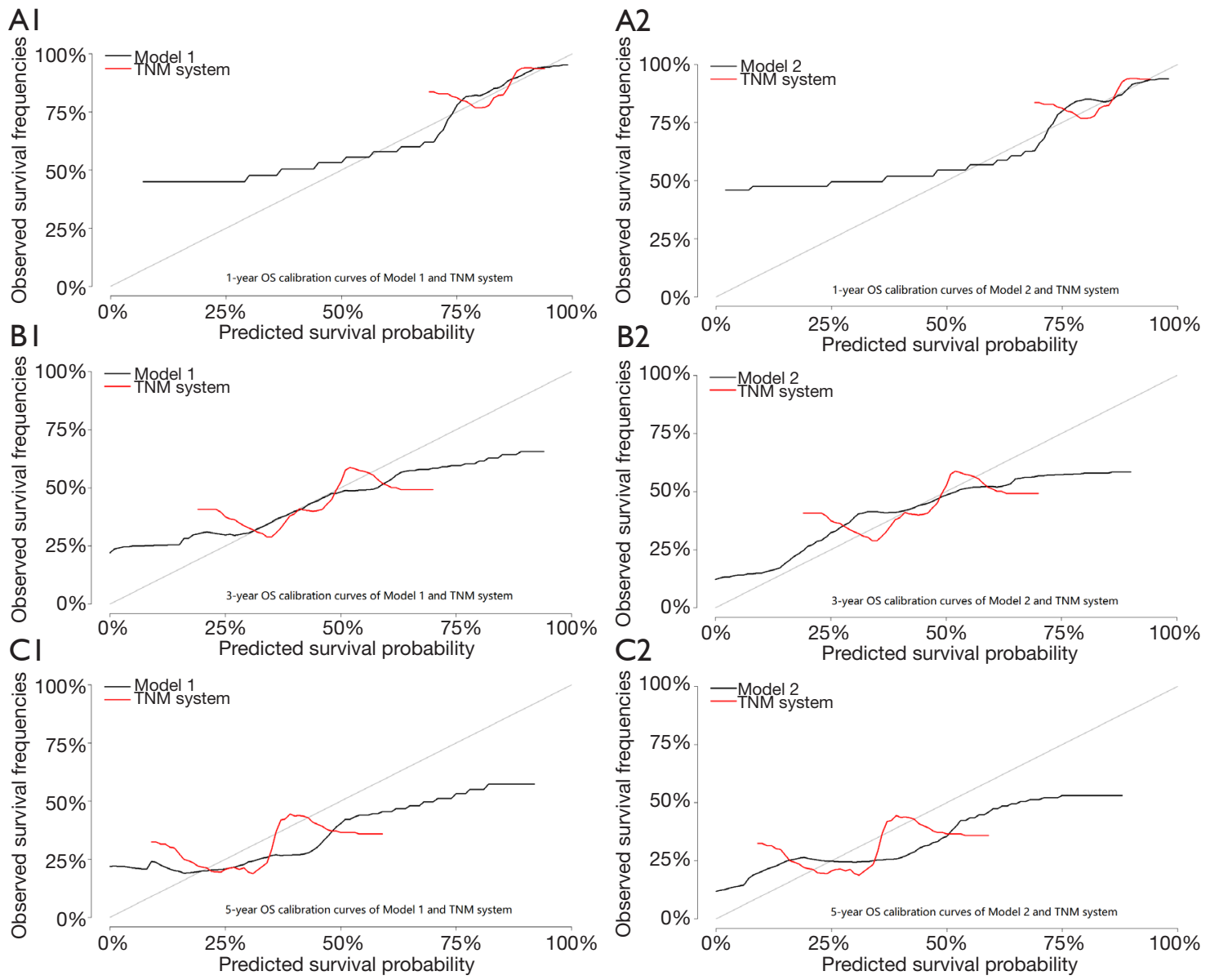

Figure 9 The calibration curve of the TNM staging system compared with model 1 and model 2 for predicting patient survival of (A1, A2) 1-, (B1, B2) 3- and (C1, C2) 5-year OS in the primary cohort after 1,000 bootstrap internal validation. TNM, tumor node metastasis; OS, overall survival.

At the same time, the research results of Japanese scholars Suzuki et al. (14) also show that higher NLR and PLR are significantly correlated with poor median and 2-year OS (NLR: 14.9 vs. 17.8 months, $29 \%$ vs. $31 \%$; $\mathrm{P}=0.026$; PLR: 14.8 vs. 18.9 months, $24 \%$ vs. $37 \% ; \mathrm{P}=0.009)$.

Shi et al. (12) revealed that NLR (HR $=2.46,95 \%$ CI: $1.508-4.011, \mathrm{P}<0.001)$ and PLR $(\mathrm{HR}=2.086,95 \%$ CI: $1.279-3.402, \mathrm{P}=0.003)$ are independent factors for predicting the survival of patients with pulmonary large cell neuroendocrine carcinoma (LCNEC). Like SCLC, as a highly malignant neuroendocrine tumor, the results also support the reliability of our study. But their research has an obvious drawback, their data lack some important clinical treatment data, such as radiotherapy and chemotherapy information, and these treatments are of great significance to this part of patients.
In addition, the collinear relationship between NLR and PLR is not clear in some studies. If both are included in the multivariate study, it is easy to ignore the prognostic significance of one of them. Therefore, we suggest that NLR and PLR should be modeled separately. Shi et al. (12) made it clear that there is a linear relationship between NLR and PLR, but still included them into multivariate regression at the same time, resulting in the loss of significant meaning in PLR, while Suzuki et al. (14) modeled the two separately, retaining their respective significant significance, and we more agree with the latter.

Some studies have shown that NLR and PLR have different prognostic significance in different stages, and high NLR and high PLR values were significantly associated with higher tumor stages. Therefore, we did a subgroup analysis according to the tumor stage, and the 
results showed that the elevated NLR and PLR had poor prognosis in each stage.

Our results may also give some enlightenment to clinicians. Patients with higher NLR and PLR before treatment may need to individually choose drug dose or multi-mode treatment, and single treatment may not be applicable. Of course, this is only a point of view, and the specific treatment model needs to be verified by prospective clinical trials, but the choice of treatment mode should be different between the two groups of patients with elevated and decreased NLR and PLR before treatment.

The study has some limitations. It is a single-center clinical retrospective study, the establishment and validation of our models were conducted internally without external validation.

\section{Conclusions}

In patients with LS-SCLC after surgery, the elevation of preoperative NLR and PLR was associated with poor OS. NLR and PLR are cheap and readily available biomarkers that may help oncologists initially identify patients with small cell lung cancer who can benefit from immunotherapy.

\section{Acknowledgments}

We would like to thank Alessio Campisi for polishing the language of this article.

Funding: This work was supported by the National Natural Science Foundation of China (81773007); Funding for Shanghai Fostering Talents by Shanghai Municipal Human Resources and Social Security Bureau (201706); Threeyear Action Plan Project for Promoting Clinical Skills and Clinical Innovation in Municipal Hospitals (16CR2013A); and Outstanding youth programme by Shanghai Municipal Commission of Health and Family Planning (2017YQ018).

\section{Footnote}

Reporting Checklist: The authors have completed the TRIPOD reporting checklist. Available at http://dx.doi. org/10.21037/tlcr-20-997

Data Sharing Statement: Available at http://dx.doi. org/10.21037/tlcr-20-997

Conflicts of Interest: All authors have completed the ICMJE uniform disclosure form (available at http://dx.doi. org/10.21037/tlcr-20-997). The authors have no conflicts of interest to declare.

Ethical Statement: The authors are accountable for all aspects of the work in ensuring that questions related to the accuracy or integrity of any part of the work are appropriately investigated and resolved. All procedures performed in this study involving human participants were in accordance with the Declaration of Helsinki (as revised in 2013). The study was approved by the institutional review board of Shanghai Chest Hospital [No. KS(Y)1755]. Because of the study's retrospective nature, the need for written informed consent was waived.

Open Access Statement: This is an Open Access article distributed in accordance with the Creative Commons Attribution-NonCommercial-NoDerivs 4.0 International License (CC BY-NC-ND 4.0), which permits the noncommercial replication and distribution of the article with the strict proviso that no changes or edits are made and the original work is properly cited (including links to both the formal publication through the relevant DOI and the license). See: https://creativecommons.org/licenses/by-nc-nd/4.0/.

\section{References}

1. Torre LA, Bray F, Siegel RL, et al. Global Cancer Statistics, 2012. CA Cancer J Clin 2015;65:87-108.

2. Goldstraw P, Ball D, Jett JR, et al. Non-small-cell lung cancer. Lancet 2011;378:1727-40.

3. de Visser KE, Eichten A, Coussens LM. Paradoxical roles of the immune system during cancer development. Nat Rev Cancer 2006;6:24-37.

4. Kemal Y, Yucel I, Ekiz K, et al. Elevated serum neutrophil to lymphocyte and platelet to lymphocyte ratios could be useful in lung cancer diagnosis. Asian Pac J Cancer Prev 2014;15:2651-4.

5. Cannon NA, Meyer J, Iyengar P, et al. Neutrophillymphocyte and platelet-lymphocyte ratios as prognostic factors after stereotactic radiation therapy for early-stage non-small-cell lung cancer. J Thorac Oncol 2015;10:280.

6. Yodying H, Matsuda A, Miyashita M, et al. Prognostic Significance of Neutrophil-to-Lymphocyte Ratio and Platelet-to-Lymphocyte Ratio in Oncologic Outcomes of Esophageal Cancer: A Systematic Review and Metaanalysis. Ann Surg Oncol 2016;23:646-54.

7. Lv Y, Zhang S, Liu Z, et al. Prognostic value of preoperative neutrophil to lymphocyte ratio is superior 
to systemic immune inflammation index for survival in patients with Glioblastoma. Clin Neurol Neurosurg 2019;181:24-7.

8. Wang Y, Qu X, Kam NW, et al. An inflammation-related nomogram for predicting the survival of patients with nonsmall cell lung cancer after pulmonary lobectomy. BMC Cancer 2018;18:692.

9. Negoi I, Beuran M, Hostiuc S, et al. Platelet-tolymphocyte ratio and CA19-9 are simple and informative prognostic factors in patients with resected pancreatic cancer. Hepatobiliary Pancreat Dis Int 2019;18:203-5.

10. Sakamoto T, Saito H, Amisaki M, et al. Combined preoperative platelet-to-lymphocyte ratio and serum carbohydrate antigen 19-9 level as a prognostic factor in patients with resected pancreatic cancer. Hepatobiliary Pancreat Dis Int 2019;18:278-84.

11. Guo M, Li W, Li B, et al. Prognostic value of delta inflammatory biomarker-based nomograms in patients with inoperable locally advanced NSCLC. Int Immunopharmacol 2019;72:395-401.

12. Shi M, Zhao W, Zhou F, et al. Neutrophil or plateletto-lymphocyte ratios in blood are associated with poor prognosis of pulmonary large cell neuroendocrine carcinoma. Transl Lung Cancer Res 2020;9:45-54.

13. Xie D, Marks R, Zhang M, et al. Nomograms Predict Overall Survival for Patients with Small-Cell Lung Cancer Incorporating Pretreatment Peripheral Blood Markers. J Thorac Oncol 2015;10:1213-20.

14. Suzuki R, Wei X, Allen PK, et al. Prognostic Significance of Total Lymphocyte Count, Neutrophil-to-lymphocyte Ratio, and Platelet-to-lymphocyte Ratio in Limited-stage Smallcell Lung Cancer. Clin Lung Cancer 2019;20:117-23.

15. Horn L, Mansfield AS, Szczęsna A, et al. First-Line Atezolizumab plus Chemotherapy in Extensive-Stage Small-Cell Lung Cancer. N Engl J Med 2018;379:2220-9.

16. Paz-Ares L, Dvorkin M, Chen Y, et al. Durvalumab plus platinum-etoposide versus platinum-etoposide in firstline treatment of extensive-stage small-cell lung cancer (CASPIAN): a randomised, controlled, open-label, phase 3 trial. Lancet 2019;394:1929-39.

17. Amaral SR, Casal Moura M, Carvalho J, et al. Prognostic significance of neutrophil-to-lymphocyte ratio (NLR) and platelet-to-lymphocyte ratio (PLR) in non-small cell lung cancer (NSCLC) treated with immune checkpoint inhibitors. Ann Oncol 2019;30.

18. Fucà G, Galli G, Poggi M, et al. Modulation of peripheral blood immune cells by early use of steroids and its association with clinical outcomes in patients with metastatic non-small cell lung cancer treated with immune checkpoint inhibitors. ESMO Open 2019;4:e000457.

19. Pavan A, Calvetti L, Dal Maso A, et al. Peripheral Blood Markers Identify Risk of Immune-Related Toxicity in Advanced Non-Small Cell Lung Cancer Treated with Immune-Checkpoint Inhibitors. Oncologist 2019;24:1128-36.

20. Passiglia F, Galvano A, Castiglia M, et al. Monitoring blood biomarkers to predict nivolumab effectiveness in NSCLC patients. Ther Adv Med Oncol 2019;11:1758835919839928.

21. Camp RL, Dolled-Filhart M, Rimm DL. X-tile: a new bio-informatics tool for biomarker assessment and outcome-based cut-point optimization. Clin Cancer Res 2004;10:7252-9.

22. Diem S, Schmid S, Krapf M, et al. Neutrophil-toLymphocyte ratio (NLR) and Platelet-to-Lymphocyte ratio (PLR) as prognostic markers in patients with nonsmall cell lung cancer (NSCLC) treated with nivolumab. Lung Cancer 2017;111:176-81.

23. Xu J, Yang H, Fu X, et al. Prophylactic Cranial Irradiation for Patients with Surgically Resected Small Cell Lung Cancer. J Thorac Oncol 2017;12:347-53.

24. Yang H, Xu J, Yao F, et al. Analysis of unexpected small cell lung cancer following surgery as the primary treatment. J Cancer Res Clin Oncol 2018;144:2441-7.

25. Zhang C, Yang H, Zhao H, et al. Clinical outcomes of surgically resected combined small cell lung cancer: a twoinstitutional experience. J Thorac Dis 2017;9:151-8.
Cite this article as: Chen C, Yang H, Cai D, Xiang L, Fang W, Wang R. Preoperative peripheral blood neutrophilto-lymphocyte ratios (NLR) and platelet-to-lymphocyte ratio (PLR) related nomograms predict the survival of patients with limited-stage small-cell lung cancer. Transl Lung Cancer Res 2021;10(2):866-877. doi: 10.21037/tlcr-20-997 
Supplementary

Table S1 The regimens of adjuvant chemotherapy

\begin{tabular}{lc} 
Chemotherapy agent & 288 \\
\hline Platinum + VP 16 & 3 \\
Platinum + Docetaxel & 2 \\
Platinum + Paclitaxel & 1 \\
Platinum + Gemcitabine & 2 \\
Platinum + Vinorelbine & 1 \\
Platinum + VP 16 + IFO & 2
\end{tabular}

$\mathrm{PCl}$, prophylactic cranial irradiation; VP-16, etoposide; IFO, ifosfamide 